\title{
Ci possiamo squagliare?
}

\author{
Paolo D'Achille
}

PUBBLICATO: 01 AGOSTO 2019

\section{Quesito:}

Sono pervenute richieste di chiarimento sull'uso del verbo squagliare (squagliarsi nella forma riflessiva), che a qualcuno sembra dialettale.

\section{Ci possiamo squagliare?}

I 1 verbo squagliare è registrato, senza alcuna marca particolare, in tutti i principali dizionari italiani, col significato di 'sciogliere, liquefare, spec. per effetto del calore' (GRADIT, che lo data al sec. XIII; concorda lo Zingarelli 20I9, che indica il I268 come anno della prima attestazione). Entrambi i dizionari considerano squagliare formato da quagliare 'coagularsi' con il prefisso privativonegativo s-. L'etimo è senz'altro convincente sul piano semantico (l'azione di 'sciogliere' si può considerare contraria a quella di 'solidificare'), ma pone problemi sotto altri aspetti: anzitutto nei nostri due dizionari quagliare ha una datazione posteriore (av. I465 per GRADIT; av. I580 per lo Zingarelli), ma in questo caso soccorre il TLIO, che riporta esempi di quaglare, quaglato in testi trecenteschi siciliani (la grafia antica $g l$ corrisponde alla moderna $g l i$ ); inoltre è un verbo intransitivo, tanto che l'antonimo non sarebbe squagliare ma piuttosto il riflessivo squagliarsi; infine, almeno nel senso proprio, è marcato come regionale (centromeridionale per il GRADIT, centrale e meridionale per lo Zingarelli). Le attestazioni piu antiche appena citate ne confermano l'origine locale.

Ora, quagliare è da considerare un allòtropo (così si indicano, nella storia della lingua, due o più parole che derivano da una stessa base) di altri due verbi che condividono il medesimo etimo, il latino coagulare, e sono affini anche per significato: appunto coagulare, parola di tradizione dotta, attestata già nel I288 (Zingarelli; TLIO), e cagliare, parola di tradizione diretta, che il GRADIT data av. I49I e lo Zingarelli av. I336, datazione che il corpus OVI sembra confermare, grazie all'esempio in un sonetto di Cenne della Chitarra: "Intorno a questo sianovi gran bagli / di villan scapigliati e gridatori / de' qual' [sic: l'apostrofo indica l'apocope della desinenza plurale $-i$, possibile nell'italiano antico] risolvan si fatti sudori / che turben l'aire si che mai non cagli". Una maggiore differenza semantica si ha tra i nomi corrispondenti: da cagliare, infatti, deriva caglio, che ha un'accezione tecnica ('sostanza acida di natura animale o anche vegetale o chimica che si aggiunge al latte per farlo coagulare al fine di ottenerne formaggio', GRADIT) sconosciuta a coagulo, che ha un significato più ampio e generico oppure indica un 'grumo solido o rappreso di sangue'; quaglio si può considerare variante antica o regionale di caglio.

S e quagliare, rispetto a cagliare e a coagulare (entrambi usati pure come transitivi), mantiene effettivamente tuttora un sentore regionale, almeno nel senso proprio (in quello figurato di 'concludere', 'arrivare al termine', usato soprattutto in frasi negative, è invece di uso generale), non cosi dovrebbe dirsi di squagliare. In effetti, mentre quagliare è registrato nel Vocabolario della Crusca solo a partire dalla terza edizione (I69I), squagliare vi compare sin dalla prima edizione (I6I2), se pure sempre con quest'unico esempio: "Ma voi, Madonna, della mia travaglia, Che si mi squaglia, prendavi mercede". Si tratta di due versi di Guido delle Colonne, un poeta federiciano, che ci riporta in Sicilia. In effetti, quella di Guido è la prima attestazione del verbo anche nel corpus OVI, che però aggiunge due esempi trecenteschi toscani, dalle Rime del Cavalca e dalla Bibbia volgarizzata. Come documenta 
anche la voce del GDLI, il verbo è stato poi usato, in varie accezioni, da autori provenienti un po' da tutte le regioni, tanto che lo si deve certamente considerare una parola italiana.

Come si spiega allora il dubbio, posto nei quesiti che ci sono pervenuti (che provengono da Roma, da Formia e da Messina), che si tratti di una voce dialettale, da sostituire con lo standard sciogliere? È verosimile che questa percezione si leghi alla maggior frequenza dell'uso del verbo in area centromeridionale (coerente con le prime attestazioni sia di squagliare sia di quagliare, che, come si è visto, sono siciliane). Probabilmente l'impressione è rafforzata dal fatto che il verbo, per certe sue caratteristiche fonetiche, ha - rispetto a sciogliere - una maggiore carica espressiva, che non ne ha consentito usi estensivi (una società si può sciogliere ma non squagliare) e che si rileva soprattutto nel riflessivo squagliarsi, più adatto di sciogliersi per dare l'idea di una liquefazione lenta, progressiva e non totale, come per es. quella dei gelati; pensiamo anche al derivato squaglio che nella locuzione squaglio di cioccolato, registrata nel GRADIT come romanesca, indica la cioccolata in tazza.

Inoltre squagliarsi, accanto al valore riflessivo, ha anche i significati, propri dell'uso colloquiale, di 'sudare abbondantemente' (mi sto squagliando dal caldo), di 'intenerirsi, sdilinquirsi' (quando parla lei, lui si squaglia) e di 'andarsene rapidamente, spec. di nascosto'. L'ultimo significato, in particolare, è registrato nel 1905 nel Dizionario moderno di Alfredo Panzini: "Squagliarsi: propr. struggersi. Familiarmente non farsi più vedere, scomparire, ma con speciale senso di chi va via per non rispondere delle proprie azioni. Voce romanesca in tale senso, estesa nel giornalismo e nell'uso a tutta Italia" (cfr. DELI, dove si segnala inoltre che esempi in dialetto di Pascarella e di Trilussa sono riportati nel Vocabolario romanesco di Gennaro Vaccaro, Roma, Romana Libri Alfabeto, I97I, s.v. squajasse). Lo stesso significato ha anche squagliarsela (in cui al riflessivo si aggiunge un altro pronome atono, la, che non ha un riferimento nel contesto: si parla in casi del genere di verbi procomplementari), che oggi in tal senso pare anzi più diffuso di squagliarsi e che è anch'esso d'uso colloquiale.

È possibile anticipare di qualche anno i più antichi esempi del significato di 'andare via di nascosto': per squagliarsi dal r905 di Panzini (DELI) possiamo risalire alla fine dell'Ottocento ("Il cavaliere della signorina, con galanteria tutta yankee, americanamente 'si squaglio', lasciando la gentile donnina nell'imbarazzo", in "Giornale delle donne", ı891, p. 39; lo slittamento semantico si intravede già in questo passo di Camillo Vacani, Storia delle campagne e degli assedi deglitaliani in Ispagna..., vol. III, Milano, Imperiale Regia Stamperia, I823, p. I03: "L'armata spagnuola poco dopo incalzata sempre più si squaglió e si disgiunse"); per squagliarsela dal 1956 (GRADIT) ai primi del secolo ("L'uditorio, come da noi ai concerti, piano piano se la squaglia"; Scipione Borghese, Dal mio taccuino. Ricordi di un viaggio in Asia, in "La Rassegna nazionale", I25, I902, p. 224).

Va segnalato, per concludere, che in Toscana, non solo per rendere questo significato invece di squagliarsi, squagliarsela si usa piuttosto svignarsela, ma che anche in senso proprio squagliare non è d'uso comune: nell'Atlante Linguistico Toscano (ALT), nelle risposte alla domanda "sciogliersi (della neve)", il nostro verbo risulta concentrato nel Grossetano meridionale e all'Isola d'Elba, mentre l'alternativa a sciogliere è piuttosto struggere.

In definitiva, l'uso di squagliare nel senso di 'sciogliere' è da considerare certamente corretto, perché si tratta di una parola italiana a tutti gli effetti. La percezione di un'origine dialettale o comunque regionale del verbo risulta tutt'altro che infondata sul piano storico e trova riscontro tuttora nella sua maggiore diffusione in area centromeridionale. Ma questa non è una buona ragione per censurarne l'uso.

Visto che questa risposta è l'ultima prima della pausa estiva, aggiungiamo due citazioni dalle Tragedie 
in due battute di Achille Campanile (Milano, Rizzoli, 1978), che ci sembrano adatte per salutarvi: l'umorista romano, infatti, riesce a dare al verbo squagliarsi sia il valore proprio, sia quello di'andare via precipitosamente': si tratta della battuta della Neve in Villeggiatura ("Io ai primi calori mi squaglio", p. I57) e della proposta del Secondo gelato come rimedio all'estate incipiente in Il congresso dei gelati ("Se ci squagliassimo?", p. I84).

Noi certamente, per qualche settimana, andiamo via; quanto ci squaglieremo, dipenderà dal caldo!

\section{Cita come:}

Paolo D'Achille, Ci possiamo squagliare?, "Italiano digitale", 2019, X, 2019/3 (luglio-settembre)

DOI: $10.35948 / 2532-9006 / 2020.3200$

\section{Copyright 2019 Accademia della Crusca}

Pubblicato con licenza creative commons CC BY-NC-ND 